\title{
A molecular mechanisms of regeneration in chronic tendinopathy using ultrasound-guided intratissue percutaneous electrolysis (EPI尺)
}

Volume 5 Issue I - 2017

\author{
Jose Manuel Sanchez-lbanez \\ CEREDE Sports Injury Clinic, Spain
}

Keywords: chronic tendon injury, clinical symptoms, nontendon cells, tendon stem cells, electrolysis

Abbreviations: SP, substance P; PRP, platelet rich plasma; ECM, extracellular matrix; VEGFR-2, vascular endothelial growth factor receptor 2; TSCs, tendon stem cells; MMPs, matrix metalloproteinase; PPAR- $\gamma$, peroxisome proliferator-activated receptor gamma; VISA-P, victorian institute of sport assessmentpatella; MMP-3, matrix metalloproteinase-3

\section{Review article}

Chronic tendon injury, or tendinopathy, refers to the clinical symptoms including pain, focal tendon tenderness, decreased strength and movement in affected tendons. Approximately $30 \%$ of general practice consultations for musculoskeletal pain are related to tendon disorders. ${ }^{1}$ Tendon injury can affect people of all ages, and can impair the activity of young and old adults in their work environment or sports activities. In summary, tendon disorders are common, have a substantial effect on quality of life and represent an important economic burden on healthcare systems. ${ }^{2}$

Tendinopathy can be identified by the following histological characteristics: collagen fibril disorganization, increased proteoglycan and glycosaminoglycan content and increased non-collagenous ECM, hypercellularity and neovascularization. ${ }^{3}$

These cellular and molecular changes modify the mechanical properties of tendon and cause pain. Because the pathogenesis of tendinopathy is not fully understood, different hypotheses have been proposed, including degeneration and failed healing. ${ }^{4}$ Tendinopathies are in the main accompanied by an excessive nociceptive signalling from the tendon, causing pain and restricted mobility. Mechanisms driving these structural and neurological changes are not fully understood. A more recent theory ascribes part of the tendinosis changes to an increased production of biochemical agents, such as substance $\mathrm{P}(\mathrm{SP})$ and NMDAR1 glutamate receptor. ${ }^{5}$

Chronic tendon injuries are facilitated by many extrinsic and intrinsic factors. Common intrinsic risk factors for tendon disorders include sex, age and diseases such as type 2 diabetes mellitus and obesity. Genetic predisposition might also influence risk of tendon injuries. ${ }^{6}$ The main recognized extrinsic factor for tendon injury is abnormal loading on tendons, which is linked to physiological exercise, sport and specific work settings. Tendinopathy is thought to result from repetitive abnormal mechanical loading, whereas acute tendon injury often results after one isolated overloading event. ${ }^{7}$ Aberrant mechanical stimulation induces the production of biological factors, including metalloproteinases, growth factors and prostaglandins, which can all lead to extracellular matrix (ECM)

\begin{abstract}
Correspondence: Jose Manuel Sánchez-lbáñez, Sports Rehabilitation Center (CEREDE), Barcelona Clinic, Spain,, Tel 349345235I0,Email drsanchez@cerede.es
\end{abstract}

Received: January 10, 2017 | Published: January 31, 2017

remodelling defects. ${ }^{8}$ Moreover, excessive mechanical loading has been proposed to cause aberrant differentiation of Tendon stem cells (TSCs) into nontendon cells. ${ }^{9}$

The primary goal of tendinopathy treatment is to reduce pain, mainly through the use of topical or systemic antiinflammatory drugs, whereas surgical techniques aim to repair ruptured tendons. ${ }^{10}$ Independently of surgical procedures or nonsurgical management of tendon injury, rerupture often occurs because scarring results in weakened tendon tissue. ${ }^{11}$ For both chronic and acute tendon injuries, exercisebased rehabilitation is indicated. ${ }^{12}$ The use of autologous growth factors is another therapeutic approach that is gaining in popularity for the treatment of tendon injury. Plateletrich plasma (PRP) is a blood derivative containing high levels of growth factors, known to promote tissue healing. ${ }^{13}$ Extracorporeal shockwave therapy has demonstrated some efficacy but only in calcified tendinitis of the shoulder; lessconventional procedures, such as phonophoresis, therapeutic ultrasonography or lowlevel laser therapy, are other options for the treatment of tendon injury. ${ }^{14}$

In recent years, the intratissue percutaneous electrolysis (EPI®) technique has become more relevant in the scientific literature given the good results yielded in the treatment of patellar degenerative tendinopathy in comparison to other previous conservative treatments. This technique (Figure 1), created by Sánchez-Ibáñez and who have over 15years' experience in its use, uses a flow of cathodic current directed exclusively to the area of degenerated tendon through an ultrasound-guided needle that brings about an organic reaction that leads to rapid regeneration of the degenerated tendón. The application of ultrasound-guided EPI ${ }^{\circledR}$ technique produces a non-thermal electrochemical reaction centered on degenerated tissue (tendinosis). 
This leads to a controlled local inflammatory reaction that leads to the regeneration of damaged tissue. ${ }^{15-18}$

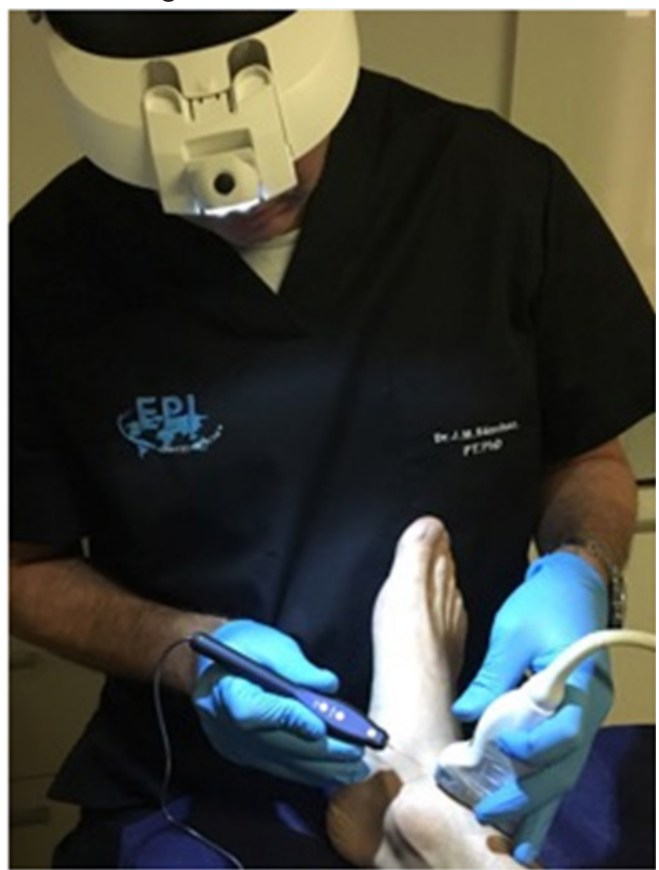

Figure I Treatment of tendinopathy with EPI ${ }^{\circ}$ device (EPI Advanced S.L. Barcelona, Spain).

In experimental studies with human tendon injury, there has been a disproportionate expression of certain cytokines and matrix metalloproteinase (MMPs), prostaglandin E2 (PGE2), interleukin-6 (IL-6) and interleukin-1b (IL-1b). IL-1b in turn increases the production of matrix metalloproteinase-1 (MMP-1), matrix metalloproteinase-3 (MMP-3) and prostaglandin E2 (PGE-2). ${ }^{19}$ A recent experimental study by Sánchez-Ibáñez JM and co-wokers (2014) showed that with the use of EPI ${ }^{\circledR}$ technique in patellar tendinopathy increase of antiinflammatory proteins, like peroxisome proliferator-activated receptor gamma (PPAR- $\gamma$ ). These proteins play a key role in the inhibition of expression of proinflammatory molecules secreted by macrophages, such as tumor necrosis factor alpha (TNF- $\alpha$ ), IL-6 and IL-1 $\beta$, thus producing in the treated tissue a highly beneficial molecular response during degenerative tendinopathy. This, in turn, results in an increase of the expression of vascular endothelial growth factor (VEGF) and vascular endothelial growth factor receptor 2 (VEGFR-2), mediators responsible for angiogenesis anti-inflammatory response. The EPI ${ }^{\circledR}$ technique makes for the activation of molecular and cellular mechanisms of the tendon responsible for phagocytosis and the regeneration of degenerated tissue. ${ }^{20} \mathrm{~A}$ study of patellar tendinopathy in humans directed by Sánchez-Ibáñez $\mathrm{JM}^{18}$ to evaluate the therapeutic effects of EPI ${ }^{\circ}$ technique on the patellar tendinopathy. The results documented were good and stable with the Victorian Institute of Sport Assessment-Patella (VISA-P) score, Tegner scores and Roles and Maudsley score, and terms of clinical and functional improvement in patellar tendinopathy and providing a follow-up of $10 y e a r .{ }^{21}$

The EPI ${ }^{\circledR}$ technique achieves a much localized organic reaction in the clinical focus by using a specially designed device (Figure 2) for this purpose (EPI Advanced Medicine, Barcelona, Spain), which leads to the rapid regeneration of degenerated tissue. This leads to the production of new immature collagen fibers that become mature by means of optimal mechanical stimulus, thereby obtaining good results in the short and long-term in terms of pain and function.

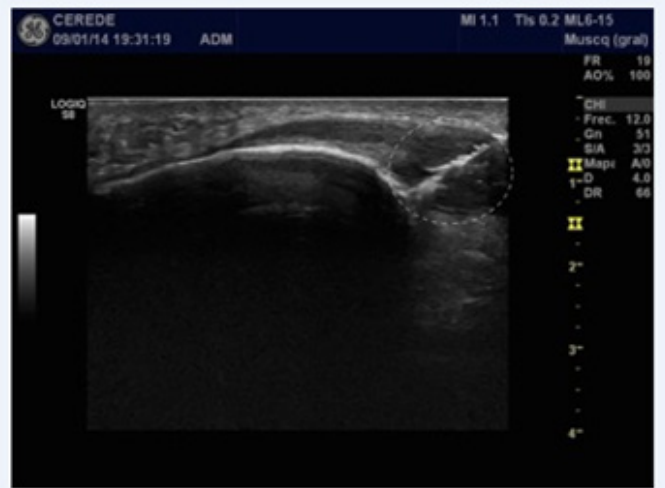

Figure 2 Hyperecoic image produced by the EPI $尺$ needle of $0,30 \mathrm{~mm}$ in the degenerative area of the tendon. This hiperecoic image corresponse to a gas density produced by the electrochemical response of the catodic flow (CF) in the degenerative extracelular matrix. Note the electrochemical reaction (white area) produced on the tip of the needle, just in the area of damaged tissue.

$E P I \circledast$ technique which leads to the rapid regeneration of degenerated tissue (Figure 3). This leads to the production of new immature collagen fibers, thereby obtaining good results in the short and long-term in terms of pain and function.

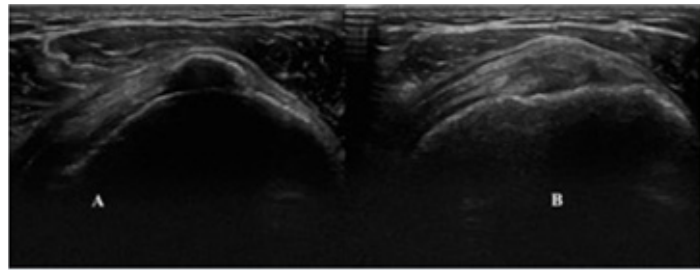

Figure 3 A) Ultrasound image in longitudinal view: Calcific tendinosis of the supraspinatus tendon with calcification; B) Three week after the EPI technique treatment. It is observed the degenerated area of the tendon that is substituted by a new connective tissue and disappearance of calcification.

\section{Acknowledgments}

None.

\section{Conflicts of interest}

Author declares there are no conflicts of interest.

\section{Funding}

None.

\section{References}

1. Kaux JF, Forthomme B, Goff CL, et al. Current opinions on tendinopathy. J Sports Sci Med. 2011;10(2):238-253.

2. American Academy of Orthpoedic Surgeons. Rotator cuff tears, Ortho Info. 2011.

3. Sharma P, Maffulli N. Tendon injury and tendinopathy: healing and repair. J Bone Joint Surg Am. 2005;87(1):187-202.

4. Magnusson SP, Langberg $\mathrm{H}$, Kjaer $\mathrm{M}$. The pathogenesis of tendinopathy: balancing the response to loading. Nat Rev Rheumatol 2010;6(5):262-268.

5. Alfredson H, Forsgren S, Thorsen $\mathrm{K}$, et al. Glutamate NMDAR1 receptors localised to nerves in human Achilles tendons: Implications for treatment? Knee Surg Sports Traumatol Arthrosc. 2001;9(2):123-126. 
6. Magnan B, Bondi M, Pierantoni S, et al. The pathogenesis of Achilles tendinopathy: a systematic review. Foot Ankle Surg. 2014;20(3):154-159.

7. Kaux JF, Forthomme B, Goff CL, et al. Current opinions on tendinopathy J Sports Sci Med. 2011;10(2):238-253.

8. Thornton GM, Hart DA. The interface of mechanical loading and biological variables as they pertain to the development of tendinosis. $J$ Musculoskelet Neuronal Interact. 2011;11(2):94-105.

9. Zhang J, Wang JH. The effects of mechanical loading on tendons-an in vivo and in vitro model study. PLoS One. 2013;8(8):e71740.

10. Childress MA, Beutler A. Management of chronic tendon injuries. Am Fam Physician. 2013;87(7):486-490.

11. Voleti PB, Buckley MR, Soslowsky LJ. Tendon healing: repair and regeneration. Annu Rev Biomed Eng. 2012;14:47-71.

12. Khan KM, Scott A. Mechanotherapy: how physical therapists prescription of exercise promotes tissue repair. $\mathrm{Br} J$ Sports Med. 2009; 43:247-252

13. Andia I, Maffulli N. Biological Therapies in Regenerative Sports Medicine Sports Med. 2016

14. Childress MA, Beutler A. Management of chronic tendon injuries. Am Fam Physician. 2016;87(7):486-490.
15. Sánchez-Ibáñez JM. Clinical evolution in the chronic pattellar enthesopathy treatment by EPI® Technique ultrasound-guided - Study of several cases in sports population. [PhD. Thesis], Universidad de León, León, Spain. 2013.

16. Sánchez-Ibáñez JM. The ultrasound-guided Intratissue Percutaneous Electrolysis (EPI $\left.{ }^{\circ}\right)$ for the Treatment of Refractory-Neovascular Patellar Tendinopathy. Int Clin Pathol J. 2017;(4(1):00080.

17. Sánchez-Ibáñez JM. Intratissue Percutaneous Electrolysis (EPI®) in the Treatment of Achilles Tendinopathy. J Nov Physiother. 2016;7:1.

18. Sànchez-Ibàñez JM, Fernández ME, Monllau JC, et al. New Treatments for Degenerative Tendinopathy, focused on the Region-Specific of the Tendon. Rheumatology. 2015;5:173.

19. Tsuzaki M, Bynum D, Almekinders L, et al. ATP modulates loadinducible IL-1beta, COX 2, and MMP-3 gene expression in human tendon cells. J Cell Biochem. 2003;89(3):556-562.

20. Abat F, Valles SL, Gelber PE, et al. Molecular repair mechanisms using the Intratissue Percutaneous Electrolysis technique in patellar tendonitis. Rev Esp Cir Ortop Traumatol. 2014;58(4):201-205.

21. Abat F, Gelber PE, Polidori F, et al. Clinical results after ultrasoundguided intratissue percutaneous electrolysis (EPI®) and eccentric exercise in the treatment of patellar tendinopathy. Knee Surg Sports Traumatol Arthrosc. 2015;23:1046-1052. 